\title{
Ecologic and Agronomic Aspects of Agrostis Capillaris L. - Trisetum Flavescens (L.) P. Beauv. Grassland Subtype
}

\author{
Ioan ROTAR ${ }^{1 *}$, Florin PĂCURAR ${ }^{1}$, Anca PLEȘA ${ }^{1}$, Roxana VIDICAN ${ }^{1}$, Miklós NAGY ${ }^{1}$ \\ Faculty of Agriculture, UASMV Cluj-Napoca, Calea Mănăștur 3-5, Cluj-Napoca, Romania; \\ * corresponding author: rotarioan52@yahoo.fr
}

Bulletin USAMV series Agriculture 71(2)/2014

Print ISSN 1843-5246; Electronic ISSN 1843-5386

DOI 10.15835/buasvmcn-agr: 10842

\begin{abstract}
Grasslands in Apuseni Mountains are used in a traditional system and several types and subtypes of grasslands were described. Because of the current grassland management and the site conditions appeared certain grassland subtypes which need to be deepen characterized and their biodiversity and agronomic value needs to be maintain. The aim of the study was to characterize Agrostis capillaris L. - Trisetum flavescens (L.) P. Beauv. grassland subtype from both, ecological and agronomic point of view. The research was unfolded within Gârda de Sus commune, on Ghețari - Poiana Călineasa Plateau. For the floristic composition's study we used the Braun-Blanquét method. The phytocenosis Agrostis capillaris L. - Trisetum flavescens (L.) P. Beauv. has been identified in 37 sites. The sites were charactherized from ecologic and agronomic point of view. This grassland subtype can be characterized as heliophytes, microtherm, mesophytes, moderate acidophilous, medium nitrophilous, medium resistant to mowing, medium resistant to stepping and medium resistant to grazing and endures an animal load of 0,81 - 1,00 LSU/ha.
\end{abstract}

Keywords: ecological factors, grasslands, phytodiversity

\section{INTRODUCTION}

Grasslands from Festuca rubra L. and Agrostis capillaris L. type in the Apuseni Mountains have evoluted toward certain types and subtypes of grasslands (Gârda, 2010). One of them is Agrostis capillaris L. with Trisetum flavescens (L.) P. Beauv. The improvement of Festuca rubra L. and Agrostis capillaris L. grasslands' trophicity determines a powerful installation of Trisetum flavescens (L.) P. Beauv. species (Coldea et al., 2012). In Europe, grasslands with Trisetum flavescens (L.) P. Beauv. have been located on the sites in the past, and were mowed two times per year and fertilized with manure (Ellenberg, 1996; Hutter et al., 2002). From the typological classification's point of view, this phytocoenosis is not described as a type or subtype, not in the nemoral or in the boreal floor (Tucra et al., 1979). The fact that Agrostis capillaris L. - Trisetum flavescens (L.) P. Beauv. phytocoenosis has been identified in many stations leads us to extend the study of these ecosystems. The aim of our study was to evaluate Agrostis capillaris L. -
Trisetum flavescens (L.) P. Beauv. grasslands, both from ecological and agronomic point of view.

\section{MATERIALS AND METHODS}

The research has been carried out in Gârda de Sus commune, between 2011-2012, where we established the study area, on Ghețari Poiana Călineasa Plateau, because it constitutes a representative area for the traditional exploitation of resources. For floristic composition we used Braun-Blanquét method, applied on $25 \mathrm{~m}^{2}$ area. Additionally, at the moment of performing the floristic relevee, by using the GPS device (Global Positioning System), data regarding the altitude (meters above sea level) and exposition (degrees $0-360^{\circ}$ ) of the relevee's research point. The distribution of identified grassland types and subtypes has been performed in several stages: marking the relevee number correspondent to the grassland on the orto-photoplan during the field activity; arranging the floristic relevees on grassland types and subtypes in Excel program; transposing these data in ArcView GIS 3.3 
programme in order to generate the map of grassland types and subtypes' distribution and establishing the area occupied by them in the studied perimeter.

Plants demands for the ecological factors (light, temperature, soil's moisture, soil's $\mathrm{pH}$ and the soil's mineral nitrogen quantity) have been embodied by species' indicator values (from 1 to 9) by Ellenberg $(1952,1992)$, upgraded by the Federal Agency of Nature Conservation Germany (Bundesamt für Naturschutz - www.floraweb.de), and adapted to the conditions of our country by Kovács (1979).

Plants resistance against mechanical disturbances, such as: mowing, stepping and grazing were embodied through indicator values (fom 1 to 9$)$ by Dierschke and Briemle (2002). Then, after Dierschke and Briemle (2002), the landuse manner's interpretation and framing into intensification classes were performed.

Taking into account the environmental factors and plant resistance on mechanical disturbances, the species were classified into specific categories in function of the environmental or disturbing factor, following the number of species in cathegory and the coverage of the cathegory. In most of cases, the coverage of a specific category can provide more accurate information than the number of species (Cristea et al., 2004). In a similar manner, the number of species in a given category is related to the total number of species in categories, without the indifferent and uncertain species. To determine the grassland's pastoral value we used the specific index of quality on a scale of 1-5 by Kovacs, 1979.

As phyto-sociological indexes, the mean abundance-dominance, the Shannon-Wiener index and the constancy have been calculated. The classification of the the grasslnads in different types and subtypes was made after Tucra et al., 1979.

\section{RESULTS AND DISCUSSIONS}

Agrostis capillaris L. - Trisetum flavescens (L.) P. Beauv. phytocoenosis was identified in 37 stations, which means a significant share $(9 \%)$ of the total studied grasslands. These grasslans are used only by mowing. In our study area this grassland subtype is found at altitudes between 1070 and $1337 \mathrm{~m}$ and occupies approximately 60 ha (Fig.1). Usually it spreads from plain lands to slopes with an angle up to $40^{\circ}$, most of the relvees being found on slopes of $10^{\circ}$. In general, this grassland is located on all of the expositions, but most commonly (35\% of stations) on eastern to southern exposition $\left(95-180^{\circ}\right)$.

Woody vegetation average cover (Tab. 1 ) is $4.46 \%$. The medium coverage of stumps is $0.88 \%$ and the rocks have $0.43 \%$. Stones have a coverage of $1.08 \%$ and the molehills have a low average coverage of $1.95 \%$. Overall medium coverage of the herbaceous vegetation is $95.92 \%$.

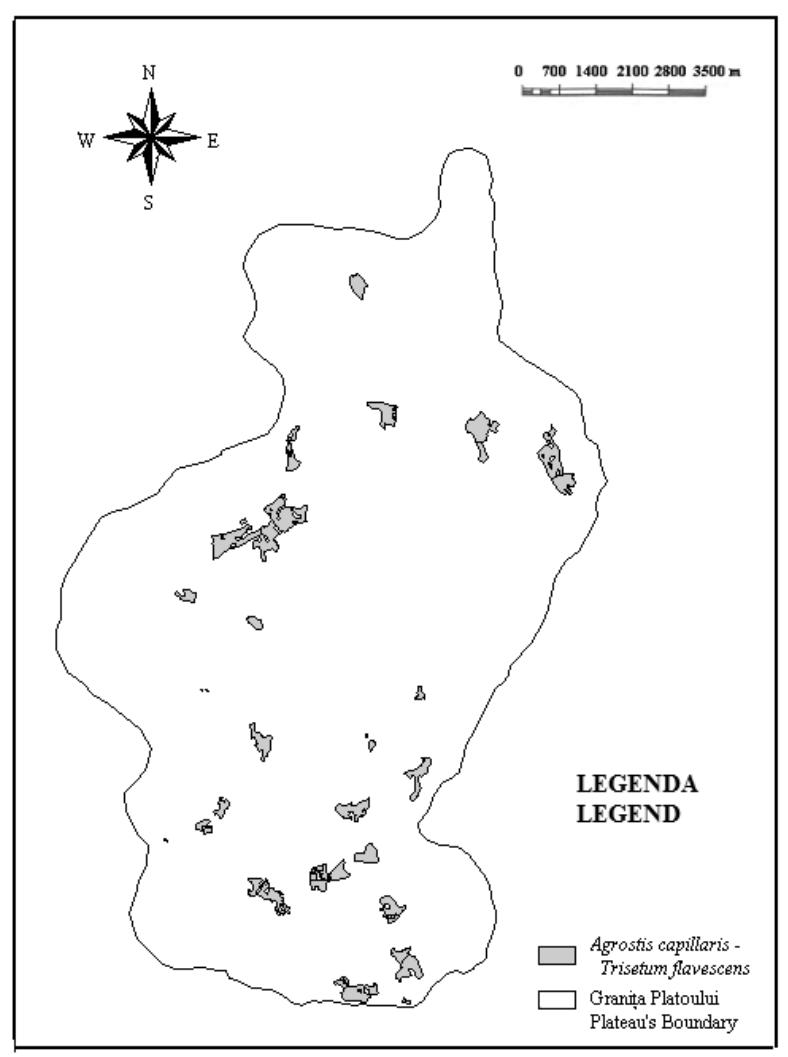

Fig. 1. Distribution of areas with the grassland subtype Agrostis capillaris L. - Trisetum flavescens

(L.) P. Beauv. on Ghețari - Poiana Călineasa Plateau

The floristic composition of Agrostis capillaris L. - Trisetum flavescens (L.) P. Beauv. varies significantly depending on the station. Poaceae sp. are present with a mean abundance-dominance (MAD) of $48.4 \%$ (Tab. 1). Agrostis capillaris L. species appears with a MAD of $24.66 \%$ and Trisetum flavescens (L.) P. Beauv. with 16.32\%. The dominant species, from Poaceae, specific for this subtype of grasslands are: Agrostis capillaris L., Festuca rubra L. and Trisetum flavescens (L.) P. Beauv. $(\mathrm{K}=\mathrm{V})$ followed by the indicator species 
Tab. 1. Stand conditions, species composition and assessment of the Agrostis capillaris L.- Trisetum flavescens (L.) P. Beauv. subtype

\begin{tabular}{|c|c|c|c|c|c|c|c|c|c|c|c|c|}
\hline & \multicolumn{4}{|c|}{ Ecological indexes } & \multicolumn{3}{|c|}{ Agronomic indexes } & \multirow{2}{*}{$\begin{array}{c}\text { Stand conditions } \\
\text { Altitude }(\mathrm{m})\end{array}$} & \multirow{2}{*}{$\frac{\overline{\mathbf{x}}}{1187,03}$} & \multirow{2}{*}{$\begin{array}{l}\mathrm{K} \\
- \\
\end{array}$} & \multirow{2}{*}{$\begin{array}{c}\mathrm{Si} \\
-\end{array}$} & \multirow{2}{*}{$\frac{\mathrm{Si}^{*} \%}{-}$} \\
\hline & & & & & & & & & & & & \\
\hline & & & & & & & & Slope $\left({ }^{\circ}\right)$ & 10,22 & - & - & - \\
\hline & & & & & & & & Exposition $\left({ }^{\circ}\right)$ & 190,19 & - & - & - \\
\hline & & & & & & & & Land use & 1,00 & - & - & - \\
\hline & & & & & & & & Wooden vegetation cover (\%) & 4,46 & - & - & - \\
\hline & & & & & & & & Stubs $(\%)$ & 0,88 & - & - & - \\
\hline & & & & & & & & Rocks $(\%)$ & 0,43 & - & - & - \\
\hline & & & & & & & & Stones (\%) & 1,08 & - & - & - \\
\hline & & & & & & & & Fallow molehills (\%) & 1,95 & - & - & - \\
\hline & & & & & & & & Moss (\%) & 1,08 & - & - & - \\
\hline & & & & & & & & General cover $(\%)$ & 95,92 & - & - & - \\
\hline $\mathrm{L}$ & $\mathrm{T}$ & $\mathrm{M}$ & $\mathrm{pH}$ & $\mathrm{N}$ & MR & GR & STR & Species & MAD & $\mathrm{K}$ & $\mathrm{Si}$ & $\mathrm{Si}^{*} \%$ \\
\hline- & - & - & - & - & - & - & - & Poaceae & 48,04 & - & - & - \\
\hline 7 & $\mathrm{x}$ & $\mathrm{x}$ & 4 & 4 & 6 & 5 & 5 & Agrostis capillaris L. & 24,66 & $\mathrm{~V}$ & 3 & 73,99 \\
\hline $\mathrm{x}$ & $\mathrm{x}$ & $\mathrm{x}$ & 5 & $\mathrm{x}$ & 7 & 5 & 5 & Anthoxanthum odoratum L. & 0,62 & III & 1 & 0,62 \\
\hline 8 & 5 & 5 & $\mathrm{x}$ & 4 & 7 & 7 & 7 & Cynosurus cristatus L. & 0,42 & III & 3 & 1,26 \\
\hline 7 & $\mathrm{x}$ & 5 & $\mathrm{x}$ & 6 & 8 & 4 & 6 & Dactylis glomerata L. & 0,35 & II & 5 & 1,76 \\
\hline 8 & $\mathrm{x}$ & 6 & $\mathrm{x}$ & 6 & 6 & 4 & 6 & Festuca pratensis Huds. & 0,50 & II & 5 & 2,50 \\
\hline $\mathrm{x}$ & $\mathrm{x}$ & 5 & $\mathrm{x}$ & $\mathrm{x}$ & 9 & 7 & 6 & Festuca rubra L. & 4,97 & $\mathrm{~V}$ & 3 & 14,90 \\
\hline 7 & $\mathrm{x}$ & $\mathrm{x}$ & $\mathrm{x}$ & 5 & 7 & 5 & 4 & Trisetum flavescens (L.) P. Beauv. & 16,32 & $\mathrm{~V}$ & 4 & 65,27 \\
\hline- & - & - & - & - & - & - & - & Cyperaceae and Juncaceae & 1,01 & - & - & - \\
\hline 7 & 4 & 7 & 3 & 4 & - & - & - & Carex leporina L. & 0,01 & I & $\mathrm{x}$ & 0,00 \\
\hline 7 & 4 & $\mathrm{x}$ & 4 & 4 & 4 & 5 & 5 & Carex pallescens L. & 0,01 & $\mathrm{I}$ & $\mathrm{x}$ & 0,00 \\
\hline 2 & 5 & 5 & 6 & 5 & - & - & - & Carex sylvatica Huds. & 0,47 & $\mathrm{I}$ & $\mathrm{x}$ & 0,00 \\
\hline 8 & 5 & 7 & 4 & 4 & 4 & 7 & 6 & Juncus effusus L. & 0,47 & $\mathrm{I}$ & $\mathrm{x}$ & 0,00 \\
\hline 7 & $\mathrm{x}$ & 4 & 3 & 2 & 5 & 5 & 5 & Luzula campestris (L.) DC. & 0,05 & $\mathrm{I}$ & $\mathrm{x}$ & 0,00 \\
\hline- & - & - & - & - & - & - & - & Fabaceae & 10,08 & - & - & - \\
\hline 7 & $\mathrm{x}$ & 4 & 7 & 3 & 6 & 4 & 4 & Lotus corniculatus L. & 2,14 & $\mathrm{~V}$ & 4 & 8,57 \\
\hline 7 & $\mathrm{x}$ & $\mathrm{x}$ & $\mathrm{x}$ & $\mathrm{x}$ & 7 & 4 & 4 & Trifolium pratense L. & 2,38 & $\mathrm{~V}$ & 4 & 9,51 \\
\hline 8 & $\mathrm{x}$ & 5 & 6 & 6 & 8 & 8 & 8 & Trifolium repens L. & 4,30 & IV & 4 & 17,19 \\
\hline 7 & 5 & 5 & $\mathrm{x}$ & $\mathrm{x}$ & 6 & 1 & 2 & Vicia cracca L. & 0,98 & IV & 2 & 1,96 \\
\hline- & - & - & - & - & - & - & - & Plants of $O B F$ & 45,15 & - & - & - \\
\hline 5 & 5 & 4 & 7 & 3 & 7 & 4 & 5 & Achillea distans Waldst. \& Kit. ex Willd. & 1,18 & II & $\mathrm{x}$ & 0,00 \\
\hline 8 & $\mathrm{x}$ & 4 & $\mathrm{x}$ & 5 & 7 & 4 & 5 & Achillea millefolium $\mathrm{L}$. & 0,47 & III & 2 & 0,95 \\
\hline 6 & 4 & 5 & 6 & 6 & & & & Alchemilla vulgaris L. & 10,47 & $\mathrm{~V}$ & 2 & 20,93 \\
\hline 6 & 4 & 6 & 8 & 5 & 3 & 7 & 3 & Astrantia major L. & 0,68 & II & $\mathrm{x}$ & 0,00 \\
\hline 8 & 6 & 5 & 7 & 5 & 5 & 2 & 2 & Campanula patula L. & 0,30 & III & $\mathrm{x}$ & 0,00 \\
\hline 8 & 4 & 5 & $\mathrm{x}$ & 6 & 6 & 6 & 6 & Carum carvi L. & 0,28 & III & 2 & 0,57 \\
\hline 7 & 4 & 5 & 5 & 4 & 5 & 2 & 2 & Centaurea phrygia L. & 4,05 & $\mathrm{~V}$ & $\mathrm{x}$ & 0,00 \\
\hline 6 & 5 & 5 & 8 & 5 & & & & Cirsium erisithales (Jacq.) Scop. & 0,51 & II & $\mathrm{x}$ & 0,00 \\
\hline 6 & 5 & 6 & 7 & $\mathrm{x}$ & $\overline{5}$ & 9 & 3 & Colchicum autumnale L. & 7,89 & IV & $\mathrm{x}$ & 0,00 \\
\hline 7 & 5 & 5 & 6 & 5 & 6 & 2 & 2 & Crepis biennis L. & 0,95 & IV & $\mathrm{x}$ & 0,00 \\
\hline 6 & $\mathrm{x}$ & $\mathrm{x}$ & $\mathrm{x}$ & 4 & 5 & 6 & 5 & Euphrasia rostkoviana Hayne & 0,42 & II & $\mathrm{x}$ & 0,00 \\
\hline 8 & $\mathrm{x}$ & 6 & 3 & 2 & 3 & 3 & 3 & Hypericum maculatum Crantz & 1,36 & IV & $\mathrm{x}$ & 0,00 \\
\hline 7 & $\mathrm{x}$ & 5 & 5 & 5 & 7 & 7 & 7 & Leontodon autumnalis L. & 1,44 & III & 1 & 1,44 \\
\hline 7 & $\mathrm{x}$ & 4 & $\mathrm{x}$ & 3 & 6 & 3 & 4 & Leucanthemum vulgare Lam. & 0,82 & IV & 1 & 0,82 \\
\hline 7 & $\mathrm{x}$ & 6 & 7 & 7 & 5 & 3 & 2 & Pimpinella major (L.) Huds. & 1,16 & IV & 1 & 1,16 \\
\hline 6 & $\mathrm{x}$ & $\mathrm{x}$ & $\mathrm{x}$ & $\mathrm{x}$ & 7 & 6 & 6 & Plantago lanceolata L. & 0,38 & III & 2 & 0,76 \\
\hline 7 & $\mathrm{x}$ & 4 & 7 & 3 & 4 & 8 & 8 & Plantago media L. & 0,34 & III & 1 & 0,34 \\
\hline 8 & 6 & 3 & 8 & 2 & 4 & 4 & 4 & Polygala comosa Schkuhr & 0,12 & II & $\mathrm{x}$ & 0,00 \\
\hline 6 & $\mathrm{x}$ & $\mathrm{x}$ & $\mathrm{x}$ & 2 & 8 & 4 & 5 & Potentilla erecta (L.) Raeusch. & 0,38 & II & 1 & 0,38 \\
\hline 8 & 6 & 3 & 7 & 3 & 6 & 5 & 4 & Ranunculus bulbosus L. & 0,59 & III & $\mathrm{x}$ & 0,00 \\
\hline 7 & 5 & $\mathrm{x}$ & $\mathrm{x}$ & 3 & 5 & 8 & 3 & Rhinanthus minor L. & 1,49 & IV & $\mathrm{x}$ & 0,00 \\
\hline 8 & $\mathrm{x}$ & $\mathrm{x}$ & $\mathrm{x}$ & 6 & 6 & 4 & 2 & Rumex acetosa L. & 1,78 & IV & $\mathrm{x}$ & 0,00 \\
\hline 8 & 5 & 3 & 8 & 3 & 5 & 3 & 4 & Scabiosa columbaria L. & 0,31 & II & $\mathrm{x}$ & 0,00 \\
\hline 6 & $\mathrm{x}$ & 4 & 4 & 3 & 4 & 5 & 5 & Stellaria graminea L. & 0,24 & III & $\mathrm{x}$ & 0,00 \\
\hline 7 & 6 & 8 & $\mathrm{x}$ & 8 & 6 & 4 & 4 & Symphytum officinale L & 0,11 & II & 1 & 0,11 \\
\hline 7 & $\mathrm{x}$ & 5 & $\mathrm{x}$ & 7 & 8 & 7 & 7 & Taraxacum officinale F.H.Wigg & 1,74 & IV & 2 & 3,49 \\
\hline 8 & $\mathrm{x}$ & 4 & $\mathrm{x}$ & 1 & 4 & 4 & 4 & Thymus pulegioides L. & 0,11 & II & $\mathrm{x}$ & 0,00 \\
\hline 9 & 3 & 7 & 6 & 5 & 5 & 7 & 2 & Trollius europaeus L. & 1,69 & II & $\mathrm{x}$ & 0,00 \\
\hline 7 & 4 & $\mathrm{x}$ & 7 & 6 & 3 & 7 & 2 & Veratrum album L. & 0,70 & II & $\mathrm{x}$ & 0,00 \\
\hline 6 & $\mathrm{x}$ & 5 & $\mathrm{x}$ & $\mathrm{x}$ & 7 & 6 & 6 & Veronica chamaedrys L. & 0,45 & II & $\mathrm{x}$ & 0,00 \\
\hline 7 & $\mathrm{x}$ & 4 & 3 & 2 & 4 & 2 & 2 & Viola canina L. & 0,12 & II & $\mathrm{x}$ & 0,00 \\
\hline 5 & 5 & $\mathrm{x}$ & $\mathrm{x}$ & $\mathrm{x}$ & 5 & 4 & 4 & Viola tricolor L. & 0,14 & II & $\mathrm{x}$ & 0,00 \\
\hline- & - & - & - & - & - & - & - & $\sum$ & - & - & - & 229,63 \\
\hline- & - & - & - & - & - & - & - & $\mathrm{P}_{4}$ & - & - & - & 2,29 \\
\hline- & - & - & - & - & - & - & - & Assessment mark & - & - & - & 50 \\
\hline
\end{tabular}

$\overline{\mathbf{X}}$ - media; K - Constancy; Si - specific index of quality; L - light, T - Temperature, M - Moisture, pH - Reaction, N - Nitrogen, MR - Resistant to mowing, GR - Resistant to grazing, STR - Resistant to stepping, MAD - Medium abundance-dominance, $\Sigma$ - Amount of SI*\%, Pv - Pastoral value. 
like Anthoxanthum odoratum L. și Cynosurus cristatus L. ( $\mathrm{K}=\mathrm{III})$ and accesory species as Festuca pratensis Huds., Dactylis glomerata L. (K = II). Other species which appears in this subtype of grassland accidentaly, determined by completely isolated conditions, are called accidental species: Deschampsia flexuosa (L.) Beauv., Elymus repens (L.) Gould, Phleum montanum K. Koch, Phleum pratense L., Poa trivialis L. etc.

Species from Cyperaceae and Juncaceae have a very reduced cover, with a MAD of $1.01 \%$ (Tab. 1), in many of the cases are missing from the canopy. Species from these botanical families are: Carex leporina L. (0,01\%), Carex pallescens L. $(0,01 \%)$, Carex sylvatica Huds. (0,47 \%), Juncus effusus L. $(0,47 \%)$ și Luzula campestris (L.) DC. (0,05 \%), all of them are considered accidental species $(\mathrm{K}=\mathrm{I})$.

Species from Fabaceae (tab. 1) are present with a MAD of $10.8 \%$. The dominant species of this family $(\mathrm{K}=\mathrm{V})$ are Trifolium pratense $\mathrm{L}$. and Lotus corniculatus L., followed by the frequent species like (K = IV) - Trifolium repens L. și Vicia cracca L.

Plants from other botanical families (OBF) are present with a MAD of $45.15 \%$. OBF species with significant presence are Alchemilla vulgaris $\mathrm{L}$. (10.47\%), Centaurea phrygia L. (4.05\%), Colchicum autumnale L. (7.89\%). The dominant species from OBF of the Agrostis capillaris L. - Trisetum flavescens (L.) P. Beauv. subtype are Alchemilla vulgaris L. and Centaurea phrygia L. $(\mathrm{K}=\mathrm{V})$, followed by frequent species (K = IV) like Colchichum autumnale L., Crepis biennis L., Hypericum maculatum Crantz, Leucanthemum vulgare Lam., Pimpinella major (L.) Huds., Rhinanthus minor L., Rumex acetosa L. and Taraxacum officinale F.H.Wigg. The indicator species of this group are $(\mathrm{K}=\mathrm{III})$ Achillea millefolium L., Campanula patula L., Carum carvi
L., Leontodon autumnalis L., Plantago lanceolata L., Plantago media L., Stellaria graminea L., etc. Accessory species (K = II) are: Achillea distans Waldst. \& Kit. ex Willd., Astrantia major L., Cirsium erisithales (Jacq.) Scop., Euphrasia rostkoviana Hayne, Polygala comosa Schkuhr, Potentilla erecta (L.) Raeusch., etc. Accidental species determined by certain factors in isolated station are: Arnica montana L., Arabis hirsuta (L.) Scop., Campanula serrata (Kit. ex Schult.) Hendrych, Carlina acaulis L., Centaurea mollis Waldst. et Kit., Doronicum orientale Hoffm., Euphorbia carniolica Jacq., Fragaria vesca L., Filipendula ulmaria (L.) Maxim., Gentianella lutescens (Velen.) J. Holub, Gymnadenia conopsea (L.) R. Br., Hieracium pilosella L., Linaria vulgaris Mill., Primula veris L., Prunella vulgaris L. etc.

Regarding the distribution of species in constancy classes, it is noted that 56 species are accidental, 10 species are indicators, 10 species are frequent and 7 species are dominant (fig. 2). There is a decrease in the number of species from the lower frequency classes to the higher classes. The trend is easily predictable and obvious, given the conditions when the biotop's limited resources maintain the abudnacy of most of the species at a relatively low level in behalf of dominant species and good competitors. This fact has been found by Cristea et al.(2004). The core of phytocoenosis consists of species from classes IV and V, total of 17 species.

The floristic diversity of this subtype is highlighted by the average value of Shannon Wiener index of 2.32. The average number of species per $25 \mathrm{~m}^{2}$ is 27.73 .

Regarding the agronomic value of grasslands, this is within the $6^{\text {th }}$ grade - grassland's cathegory is medium and endures a load of 0.81 to $1.00 \mathrm{LSU} / \mathrm{ha}$.

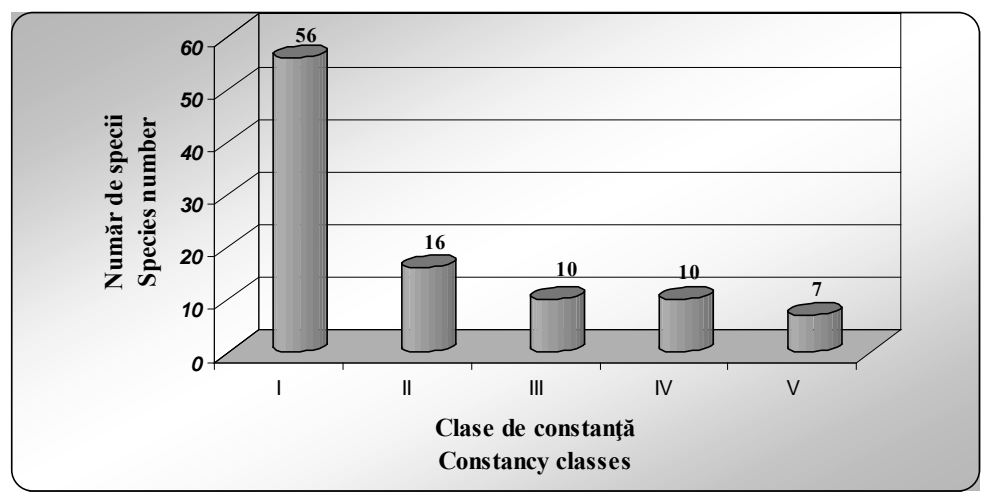

Fig. 2. Distribution on constancy classes of Agrostis capillaris L.- Trisetum flavescens (L.) P. Beauv. subtype's species 
Within this subtype 72 heliophytes species, 18 meso-heliophytes, 5 extremely-heliophytes and one heliophobic species are distinguished (Tab. 2). The same hierarchy is maintained when considering the cover of the species' classes and we can say that this subtype has a heliophytes character.

Regarding the special requirements for temperature of the species, we found out that 37 species are mesotherm and 10 are microtherme. The other categories are not represented and 52 species are indifferent to temperature (Tab. 2). Even though $69.19 \%$ is represented by the coverage of indifferent species for temperature, we state that this subtype has a character microtherme, because the microtherme species have a coverage of $18.23 \%$.
Regarding the requirement for moisture of the plants we observed that 38 species are mesophytes, 33 mesoxerophiles and 12 mesohygrophylic (Tab. 2). The same hierarchy is maintained when considering species cover and we can say that the subtype has a mesophytes character ( $44.54 \%$ coverage).

In canopy 24 neutrophilous species, 14 moderate acidophilous species, 13 alkaliphile species, 8 lightly acidophilous species and one strongly acidophilous species are present. The number of indifferent species to soil reaction is 38 (Tab. 3). Considering the coverage of species, we found out that the highest coverage have the moderate acidophilous species, followed by the lightly acidophilous species and neutrophilous with alkaliphile species. Thus, we can say that this subtype has a moderate acidophilous character. Tab. 3)

Tab. 2. Classification of Agrostis capillaris L.- Trisetum flavescens (L.) P. Beauv. subtype's species depending on demand for light, temperature and moisture

\begin{tabular}{lccccccccc}
\hline & \multicolumn{3}{c}{ Light } & \multicolumn{3}{c}{ Temperature } & \multicolumn{3}{c}{ Moisture } \\
\cline { 2 - 12 } Classes & $\begin{array}{c}\text { Species } \\
\text { no. }\end{array}$ & $\begin{array}{c}\text { Species } \\
\text { by LI } \\
\%\end{array}$ & $\begin{array}{c}\mathrm{C} \\
\%\end{array}$ & $\begin{array}{c}\text { Species } \\
\text { no. }\end{array}$ & $\begin{array}{c}\text { Species } \\
\text { by TI } \%\end{array}$ & $\begin{array}{c}\text { C } \\
\%\end{array}$ & $\begin{array}{c}\text { Species } \\
\text { no. }\end{array}$ & $\begin{array}{c}\text { Species } \\
\text { by MI } \\
\%\end{array}$ & $\begin{array}{c}\text { C } \\
\%\end{array}$ \\
\hline $1-2$ & 1 & 1,01 & 0,47 & 0 & 0,00 & 0,00 & 0 & 0,00 & 0 \\
\hline $3-4$ & 0 & 0,00 & 0,00 & 10 & 10,10 & 18,23 & 33 & 33,33 & 7,71 \\
\hline $5-6$ & 18 & 18,18 & 23,16 & 37 & 37,37 & 16,88 & 38 & 38,38 & 44,53 \\
\hline $7-8$ & 72 & 72,73 & 72,97 & 0 & 0,00 & 0,00 & 12 & 12,12 & 2,58 \\
\hline 9 & 5 & 5,05 & 1,98 & 0 & 0,00 & 0,00 & 0 & 0,00 & 0 \\
\hline $\mathrm{x}$ & 3 & 3,03 & 5,72 & 52 & 52,53 & 69,19 & 16 & 16,16 & 49,48 \\
\hline Total & 96 & - & - & 47 & - & - & 83 & - & \\
\hline
\end{tabular}

Note: C - species cover; LI - light index; TI - tempearture index; MI - moisture index; The total was calculated without indifferent and uncertain species.

Tab. 3. Classification of Agrostis capillaris L.- Trisetum flavescens (L.) P. Beauv. subtype's species depending on demand for soil's $\mathrm{pH}$

\begin{tabular}{lccc}
\hline \multirow{2}{*}{ Classes } & \multicolumn{3}{c}{ Soil's $\mathrm{pH}$} \\
\cline { 2 - 4 } & Species no. & Species by pHI $\%$ & 0,01 \\
\hline $1-2$ & 1 & 1,01 & 27,34 \\
\hline $3-4$ & 14 & 14,14 & 23,99 \\
\hline $5-6$ & 8 & 8,08 & 15,29 \\
\hline 7 & 24 & 24,24 & 2,04 \\
\hline $\mathrm{x}-9$ & 13 & 13,13 & 35,42 \\
\hline $\mathrm{u}$ & 38 & 38,38 & 0,20 \\
\hline Total & 1 & 1,01 & - \\
\hline
\end{tabular}

Note: C-species cover; pHI-pH index; u-uncertain; The total was calculated without indifferent and uncertain species. 
The phytocoenosis of this subtype includes 29 moderat nitrophilous species, 26 medium nitrophilous species, 20 nitrophobic and 10 nitrophilous (Tab. 4). The highest coverage have the medium nitrophilous species (41.83\%), followed by moderate nitrophilous species. Thus the subtype Agrostis capillaris L.- Trisetum flavescens (L.) P. Beauv. phytocoenosis has a medium nitrophilous character.

Regarding plant resistance to mowing, we found that 31 species are medium resistant, 29 are medium sensitive, 20 resistant and 2 extremely resistant. If we consider the coverage, it is found that the group of medium resistant species has the highest coverage (51.12\%), followed by the resistant species (Tab. 4). Thus, we can state that the subtype has a medium resistant character on mowing, being used in an extensive to medium intensive system.

Regarding stepping resistance we found that 34 species are medium sensitive, 23 medium resistant, 17 sensitive and 8 resistant (Tab. 5). When coverage percent is taken into account, it is found that the medium sensitive species and medium resistant species have about the same value (35.7\%, respectively $35.81 \%)$, followed by sensitive species $(12.40 \%)$ and resistant species (8.36\%). Grassland has a medium sensitive character on stepping, which shows that stepping occurs only occasionally by agricultural machinery or once during a grazing cycle.

In Agrostis capillaris L.- Trisetum flavescens (L.) P. Beauv. phytocoenosis subtype 34 species are medium sensitive to grazing, 18 species medium

Tab. 4. Classification of Agrostis capillaris L.- Trisetum flavescens (L.) P. Beauv. subtype's species depending on demand for nitrogen and mowing suitability

\begin{tabular}{lcccccc}
\hline \multirow{2}{*}{ Classes } & \multicolumn{3}{c}{ Nitrogen } & \multicolumn{3}{c}{ Mowing resistance } \\
\cline { 2 - 7 } & $\begin{array}{c}\text { Species } \\
\text { no. }\end{array}$ & $\begin{array}{c}\text { Species by NI } \\
\%\end{array}$ & C $\%$ & Species no. & $\begin{array}{c}\text { Sepcies by MI } \\
\%\end{array}$ & $\mathrm{C} \%$ \\
\hline $1-2$ & 20 & 20,20 & 3,22 & 0 & 0,00 & 0,00 \\
\hline $3-4$ & 29 & 29,29 & 37,88 & 29 & 29,29 & 5,41 \\
\hline $5-6$ & 26 & 26,26 & 41,83 & 31 & 31,31 & 51,12 \\
\hline $7-8$ & 10 & 10,10 & 3,20 & 20 & 20,20 & 30,69 \\
\hline 9 & 0 & 0,00 & 0,00 & 2 & 2,02 & 5,05 \\
\hline $\mathrm{x}$ & 14 & 14,14 & 18,18 & - & - & - \\
\hline $\mathrm{u}$ & - & - & - & 17 & 17,17 & 12,04 \\
\hline Total & 85 & - & - & 82 & - & - \\
\hline
\end{tabular}

Note: C-species cover; NI-nitrogen index; MI-mowing index; u-uncertain; The total was calculated without indifferent and uncertain species.

Tab. 5. Classification of Agrostis capillaris L. - Trisetum flavescens (L.) P. Beauv. subtype's species depending on stepping and grazing suitability

\begin{tabular}{lcccccc}
\hline \multirow{2}{*}{ Classes } & \multicolumn{3}{c}{ Stepping resistance } & \multicolumn{3}{c}{ Grazing resistance } \\
\cline { 2 - 7 } & $\begin{array}{c}\text { Species } \\
\text { no. }\end{array}$ & $\begin{array}{c}\text { Species by STI } \\
\%\end{array}$ & $\mathrm{C} \%$ & $\begin{array}{c}\text { Species } \\
\text { no. }\end{array}$ & $\begin{array}{c}\text { Species by GI } \\
\%\end{array}$ & $\mathrm{C} \%$ \\
\hline $1-2$ & 17 & 17,17 & 12,40 & 11 & 11,11 & 6,79 \\
\hline $3-4$ & 34 & 34,34 & 35,70 & 34 & 34,34 & 14,51 \\
\hline $5-6$ & 23 & 23,23 & 35,81 & 18 & 18,18 & 44,30 \\
\hline $7-8$ & 8 & 8,08 & 8,36 & 18 & 18,18 & 18,78 \\
\hline 9 & 0 & 0,00 & 0,00 & 1 & 1,01 & 7,89 \\
\hline $\mathrm{u}$ & 17 & 17,17 & 12,04 & 17 & 17,17 & 12,0351 \\
\hline Total & 82 & - & - & 82 & - & - \\
\hline
\end{tabular}

Note: C-species cover; STI-stepping index; GI-grazing index; u-uncertain; The total was calculated without indifferent and uncertain species. 
resistant, 18 resistant species, 11 sensitive and one extremely resistant species (Tab. 5). Considering the coverage, we can state that $44.3 \%$ are medium resistant species, followed by resistant (18.78\%), medium sensitive and sensitive species. In this case, the character of grassland is medium resistant to grazing, and we can conclude that the grassland is grazed 1-2 times per year.

\section{CONCLUSION}

The presence of Agrostis capillaris L.- Trisetum flavescens (L.) P. Beauv. grasslands in the study area in a high level is fully justified with their framing as subtype of Festuca rubra L. - Agrostis capillaris L.grassland type.

Agrostis capillaris L. - Trisetum flavescens (L.) P. Beauv. subtype can be characterized as heliophytes, microtherm, mesophytes, moderate acidophilous, medium nitrophilous, medium resistant to mowing, medium resistant to stepping and medium resistant to grazing.

Regarding grassland's agronomic value, this frames within the $6^{\text {th }}$ class, the grassland category is medium and can support an animal load of 0,81 $-1,00 \mathrm{LSU} / \mathrm{ha}$.

\section{REFERENCES}

1. Braun- Blanquet J. (1932). Plant Sociology, the study of plant communities, Ed. Mc-Graw - Hill Book Company, Inc. New - York and London, 31-33.

2. Coldea Gh., Oprea A., Sârbu I., Sîrbu C., Ștefan N. (2012). Les associations Vegetales de Roumanie. Presa Universitară Clujană, Cluj-Napoca.

3. Cristea V., Gafta D., Pedrotti F. (2004). Phytosociology. Presa Universitară Clujeană Printhouse.

4. Dierschke H., G. Briemle (2002). Kulturgrasland. Wiesen, Weiden und verwandte Staudenfluren, Ed. Verlag Eugen Ulmer GmbH \& Co., Stuttgart, Germania, 31-33, 205-216.

5. Ellenberg H. (1952). Wiesen und Weiden und ihre standörtliche Bewertung, Ed.Ulmer, Stuttgart.

6. Ellenberg H., Weber H. E., Düll R., Wirth Werner V., Paulissen W. D. (1992). Zeigerwerte von Pflanzen in Mitteleuropa. - Scripta geobotanica 18.

7. Ellenberg H. (1996). Vegetation Mitteleuropas mit den Alpen in ökologischer, dynamischer und historischer Sicht. 5. Auflage. Stuttgart, Ulmer. 1096 S.

8. Gârda N. (2010). The study of some mountainouslandscape elements (with special regard to grassland ecosystems in Gârda de Sus Commune, Apuseni Mountains. PhD Thesis, Faculty of Agriculture, USAMV Cluj-Napoca.

9. Huttler C.P., Briemle G., Fink C. (2002). Wiesen, Weiden und anderes Grünland. Hirzel. Germany.

10. Kovács J.A. (1979). Indicatorii biologici, ecologici şi economici ai florei pajiștilor, Redacția de propagandă tehnică agricolă, 1-50.

11. Țucra I., Kovacs A.J., Roșu C., Ciubotaru C., Chifu T., Neacșu M., Bărbulescu C., Cardașol V., Popovici D., Simtea N., Motcă Gh., Dragu I., Spirescu M. (1987). Principalele tipuri de Pajişti din R.S. Romania, Ed. București.

12. ${ }^{* * *}$ www.floraweb.de 